\section{Polymer science}

\section{Novel helical conformation in a nylon}

\section{from Edward Atkins}

X-RAY structure determinations undertaken so far on different nylons have shown that the chains crystallize in an extended planar zig-zag conformation. In the crystals, the vital amide groups (-NHCO-) participate in interchain hydrogenbonding in the plane of the zig-zag, generating hydrogen-bonded sheets which stack to form the three-dimensional crystalline structure. The properties and behaviour of the nylons examined until now all relate to this type of molecular architecture. In this issue of Nature (page 53), Fernández-Santin, Aymamí, Rodriguez-Galán, Muñoz-Guerra and Subirana describe a novel structure for a nylon derivative, poly( $\alpha$-isobutil-Laspartate) : a contracted spring-like helix, stabilized by intrachain hydrogen bonds, which exhibits a number of similarities with the well-known $\alpha$-helix found in proteins and polypeptides (see Fig.3 on page 53). This novel spring-like conformation is of interest not only for its own sake and because of the implications it has for the properties of a nylon, but also because it highlights the link nylons can provide between synthetic polymers and proteins.

The nylons or polyamides represent a spectrum of synthetic polymers in which amide groups introduce a hydrogenbonding capability into the structure. At one extreme, the amide groups occur very infrequently, and the nylons approach the conformation, behaviour and properties of polyethylene: an extended planar zig-zag chain associating with neighbouring chains through relatively weak van der Waals' interactions. Near the other end of the spectrum, where the amide groups are separated only by one or a few $\mathrm{CH}_{2}$ units, is the structure, known as nylon-2, which is chemically identical to polyglycine - a polypeptide or simple protein - in which two carbon atoms $\left(-\mathrm{CH}_{2}-\mathrm{CO}-\right)$ occur between successive nitrogen atoms in the chain. (The ultimate polyamide is nylon-1.) The bulk of the nylons used industrially have amide groups distributed with a frequency intermediate between these two extremes. A common polyamide is nylon- 6 with six carbon atoms between successive nitrogens. It might be predicted, $a$ priori, that as the frequency of amide groups increases and the nylons take on a similar chemistry to that of polypeptides and proteins, they would be capable of mimicking their biopolymeric counterparts.

In proteins, only a relatively small number of regular conformations have been found, of which the $\alpha$-helix, the 3 -fold or collagen-like helix and the $\beta$-sheet are the most prominent. These regular structures are ubiquitous in fibrous proteins (Fraser, R.D.B. \& MacRae, T.P. Conformation in Fibrous Proteins, Academic, New York; 1973) and occur as domains, connected by less regular segments, in the convoluted conformations found in globular proteins, for example myoglobin, haemoglobin and the enzymes. $\beta$-sheets are extended protein chains, hydrogen-bonded together to form sheets. They occur in many insect silks, and can be thought of as the biopolymeric analogues to the traditional nylon struc-

\section{Anaesthesia}

\section{from W. Graham Richards}

tures. Unlike the $\beta$-structures, which are stabilized by interchain hydrogenbonding, the $\alpha$-helix is a contracted springlike helix stabilized by intrachain hydrogen bonds, and is found in many keratinous tissues such as hair.

The novel structure discovered by Fernández-Satin et al. for nylon-3, which closely approaches the frequency of amide groups in proteins (variants of nylon-2 backbone), is a departure from the traditional nylon structures and represents the first of what is hoped to be a family of self-stabilized spring-like helices for nylons. These substances should exhibit behaviour and properties quite different from those of previous nylons, but similar to those of the extensively studied $\alpha$-polypeptides and $\alpha$-proteins.

Edward Atkins is Professor of Physics at the H.H. Wills Physics Laboratory, University of Bristol, Tyndall Avenue, Bristol B88 1TL.

\title{
Glycine receptor protein the solution?
}

AlCOHOL added to a fish tank containing newts will anaesthetize the newts and they will keel over unconscious. Surprisingly, a subsequent increase of the hydrostatic pressure on the water of the tank reverses this anaesthesia and the unconscious creatures revive and will swim normally under the increased pressure. This is an example of the pressure-reversal of anaesthesia which is widely observed across a huge range of species and ought to provide some clue as to the mechanism of general anaesthesia. Work described in this issue of Nature (page 56) uses this approach and comes up with some intriguing results which may implicate glycine-mediated synaptic inhibition in the action of anaesthetics.

In pharmacological terms, general anaesthesia seems to be a rather crude affair not involving very specific molecular receptors: large concentrations of anaesthetic are used and almost any gas, even an inert gas such as helium, will act as an anaesthetic if applied at sufficient pressure. A vital practical ramification of this is the narcosis suffered by divers who can be made semiconscious as a result of the anaesthetic effects of nitrogen in air when they reach depths at which the anaesthetic pressure becomes significant.

The traditional interpretation of this lack of specificity and the influence of pressure has been that the effect of anaesthetic has a relatively simple physical origin. Since anaesthetic potency correlates with solubility in fat, it has been held that the results are achieved by solubility of the gas in lipid membranes; swelling produces anaesthesia reversible by compressing the membranes. Alternatively, the hydrophobic part of a specific protein could be the site of solution.

In this week's Nature, Smith et al. provide a significant step forwards in elucidating the mechanism of anaesthesia in more precise molecular terms. The key observation is a complete difference in response to pressure between anaesthetized tadpoles and shrimps with anaesthetics chloroform, ether, ethanol, halothane and sodium pentabarbitone. Thus, whereas the relative potencies of these anaesthetics in the two species are very similar, the influence of pressure is totally opposite in the two cases: shrimps show no sign of pressure-reversal, indeed pressure can potentiate anaesthesia, while in tadpoles, the effects of anaesthetic are reversed by the application of pressure in otherwise identical circumstances.

The authors' preferred explanation of this dramatic difference derives from the fact that crustaceans, such as the freshwater shrimp, have a nervous system which is insensitive to strychnine. This implies they do not use glycine as a neurotransmitter. Thus, it is possible that the site of the solution of an anaesthetic is a hydrophobic part of the glycine receptor protein. Support for this notion comes from the fact that the pharmacology of pressure, as elucidated from pressure-induced convulsions in mammals, closely parallels that of strychnine which is believed to act on postsynaptic inhibition mediated by glycine. $\square$

W. Graham Richards is a lecturer in the Physical Chemistry Laboratory, University of Oxford, South Parks Road, Oxford OXI $3 Q Z$. 